This item was submitted to Loughborough's Research Repository by the author.

Items in Figshare are protected by copyright, with all rights reserved, unless otherwise indicated.

\title{
A new cross-correlation and constant modulus type algorithm for PAM-PSK
} signals

PLEASE CITE THE PUBLISHED VERSION

PUBLISHER

(c) IEEE

VERSION

VoR (Version of Record)

LICENCE

CC BY-NC-ND 4.0

\section{REPOSITORY RECORD}

Luo, Yuhui, and Jonathon Chambers. 2019. "A New Cross-correlation and Constant Modulus Type Algorithm for PAM-PSK Signals". figshare. https://hdl.handle.net/2134/5758. 
This item was submitted to Loughborough's Institutional Repository (https://dspace.lboro.ac.uk/) by the author and is made available under the following Creative Commons Licence conditions.

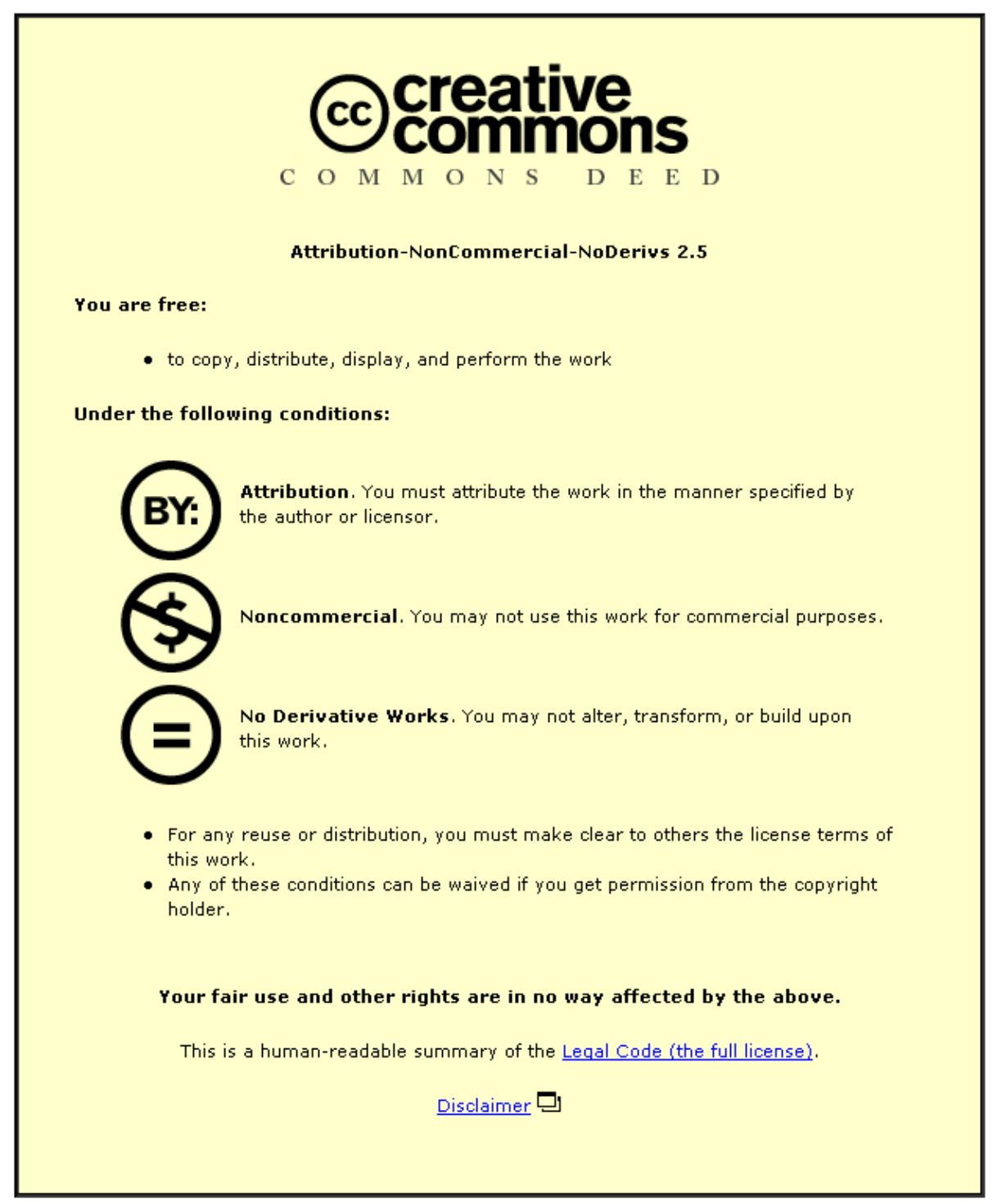

For the full text of this licence, please go to: http://creativecommons.org/licenses/by-nc-nd/2.5/ 


\title{
A NEW CROSS-CORRELATION AND CONSTANT MODULUS TYPE ALGORITHM FOR PAM-PSK SIGNALS
}

\author{
J.A. Chambers and Y. Luo \\ Centre for Digital Signal Processing Research \\ School of Physical Sciences and Engineering, King's College London \\ Strand, London WC2R 2LS, United Kingdom. Email: Yuhui.Luo@kcl.ac.uk
}

\begin{abstract}
We address the problem of blind recovery of multiple sources from their linear convolutive mixture with the cross-correlation and constant modulus algorithm. The steady state meansquared error of this algorithm is first derived to justify the proposal of a new cross-correlation and constant modulus type algorithm for PAM-PSK type non-constant modulus signals. Simulation studies are presented to support the improved steady-state performance of the new algorithm.
\end{abstract}

\section{INTRODUCTION}

In a multi-input and multi-output (MIMO) system, one of the challenging problems is to reconstruct multiple independent and identically distributed (i.i.d.) sources from their linear convolutive mixture. In spatial division multiple access (SDMA) for use in multiuser telecommunications, for example, a number $U$ of i.i.d. sources is transmitted through linear channels, which can be modeled as FIR filters of order $L$, and picked up by an array $R$ of sensors to exploit spatial diversity. A bank of space-time equalizers is employed to mitigate intersymbol interference (ISI) and interuser interference (IUI). Among various adaptive algorithms proposed, the cross-correlation and constant modulus algorithm (CC-CMA) utilizes the underlying constant modulus property of the transmitted signals and separates different signals by introducing a decorrelation penalty within the cost function, [1]. Provided that some mild conditions regarding the channel and the sources are satisfied, the $\mathrm{CC}$ CMA algorithm exhibits mean convergence to a zero-forcing solution corresponding to the retrieval of one of the multiple sources with possible delay. However, the mean-squared error (MSE) of the CC-CMA algorithm in the steady state cannot be avoided due to the stochastic gradient used in the equalizer update equation. In this paper, by applying a similar approach as [2], the steady state MSE of the CCCMA algorithm is derived. As the analytical expression indicates that the steady state MSE of the CC-CMA algorithm increases when the signal constellation is not constant modulus, a novel integration of the CC-CMA cost function together with a constellation match error penalty is proposed for Pulse Amplitude Modulation - Phase Shift Keying (PAM-PSK) type signals in a multiuser system.

\section{THE CC-CMA ALGORITHM}

At time instant $k$, the equalizer regressor is given by

$$
\mathbf{x}(k)=\left[\mathbf{x}_{1}^{T}(k) \ldots \mathbf{x}_{R}^{T}(k)\right]^{T}=\Delta^{T} \mathbf{s}(k)
$$

where $\mathbf{x}_{j}(k)=\left[x_{j}(k) \ldots x_{j}(k-N)\right]^{T}$ is the $j^{\text {th }}$ sensor output vector, $N$ is the order of the sub-equalizer, $\triangle$ is the channel convolution matrix, $\mathbf{s}(k)=\left[s_{1}(k) \ldots s_{1}(k-N-\right.$ $\left.L) \ldots s_{U}(k-N-L)\right]^{T}$ is the composite source vector, [3]. The notations $(\cdot)^{H},(\cdot)^{T}$ and $(\cdot)^{*}$ denote respectively hermitian, transpose and complex conjugate. Representing the $l^{\text {th }}$ space-time equalizer tap vector as $\mathbf{w}_{l}(k)$, its output is written as $y_{l}(k)=\mathbf{w}_{l}^{T}(k) \mathbf{x}(k)$. Define $\mathbf{h}_{l}(k)$ as the combined channel and equalizer- $l$ impulse response. The $l^{\text {th }}$ equalizer output can also be written as $y_{l}(k)=\mathbf{h}_{l}^{T}(k) \mathbf{s}(k)$. The CCCMA algorithm cost function for the $l^{\text {th }}$ equalizer is written as

$J_{l}(k)=E\left\{\left(\left|y_{l}(k)\right|^{2}-R_{2}\right)^{2}\right\}+\gamma \sum_{m=1}^{l-1} \sum_{\delta=-(N+L)}^{N+L}\left|r_{m, \delta}(k)\right|^{2}$

where $E\left\{\left(\left|y_{l}(k)\right|^{2}-R_{2}\right)^{2}\right\}$ is the constant modulus cost, $R_{2}=\frac{E\left\{|s|^{4}\right\}}{E\left\{|s|^{2}\right\}}$ is the so-called dispersion constant, $\gamma \in R^{+}$ is the mixing parameter and $r_{m, \delta}(k)=E\left\{y_{l}(k) y_{m}^{*}(k-\delta)\right\}$ is the cross-correlation between the $l^{\text {th }}$ equalizer and the $m^{\text {th }}$ equalizer output with $\operatorname{lag} \delta$. For notational convenience, we rewrite the cost function in eqn (2) as

$$
J_{l}(k)=E\left\{\left(\left|y_{l}(k)\right|^{2}-R_{2}\right)^{2}\right\}+\mathbf{r}^{T}(k) \mathbf{y}^{\prime}(k)
$$

where $\mathbf{r}(k)$ and $\mathbf{y}^{\prime}(k)$ are vectors containing the elements of $r_{m, \delta}(k)$ and $y_{m}(k-\delta)$ with different values in the indices $m$ and $\delta$, i.e., $\mathbf{r}(k)=\left[r_{1,-(N+L)}(k) \ldots \ldots r_{l-1,(N+L)}(k)\right]^{T}$ 
and $\mathbf{y}^{\prime}(k)=\left[y_{1}(k+N+L) \ldots \ldots y_{l-1}(k-N-L)\right]^{T}$. With the parameter $\lambda \in(0,1]$ controlling the length of the effective data window in the estimation, the cross-correlaton vector $\mathbf{r}(k)$ can be estimated by the following update equation.

$$
\widehat{\mathbf{r}}(k)=\lambda \widehat{\mathbf{r}}(k-1)+(1-\lambda) y_{l}(k) \mathbf{y}^{\prime *}(k)
$$

With the stochastic gradient descent method, the $l^{\text {th }}$ equalizer update equation is given by

$$
\mathbf{w}_{l}(k+1)=\mathbf{w}_{l}(k)+\mu e_{o}^{l}(k) \mathbf{x}^{*}(k)
$$

where the positive scalar $\mu$ is the step size parameter and $e_{o}^{l}(k)=\left(R_{2}-\left|y_{l}(k)\right|^{2}\right) y_{l}(k)-\frac{\dot{\gamma}}{2} \widehat{\mathbf{r}}^{T}(k) \mathbf{y}^{\prime}(k)$ is the instantaneous error signal of the $l^{\text {th }}$ equalizer.

\section{THE CC-CMA STEADY STATE MSE ANALYSIS}

We note that additive channel noise is inevitable in all practical communication models and contributes to the steady state MSE. However, in this study, as in [2], a noise-free environment is assumed since our focus is on the influence of the stochastic gradient estimator of the CC-CMA algorithm towards its steady state MSE performance. Without loss of generality, we assume that the $l^{\text {th }}$ equalizer asymptotically converges to the retrieval of the $l^{\text {th }}$ source with delay $d_{l}$, [4]. Define a priori error $e_{a}^{l}(k)$ for the $l^{\text {th }}$ equalizer at the $k^{\text {th }}$ iteration as

$$
e_{a}^{l}(k)=s_{l}(k-d)-y_{l}(k)
$$

The expression of the steady state MSE for the $l^{\text {th }}$ equalizer is shown in eqn (7) and this is the quantity that we wish to determine.

$$
M S E=\lim _{k \rightarrow \infty} E\left\{\left|e_{a}^{l}(k)\right|^{2}\right\}
$$

Before continuing, the statistical properties of the crosscorrelation estimator $\widehat{r}_{m, \delta}(k)$ after convergence of the $l^{\text {th }}$ equalizer are studied.

(a) The expectation of the cross-correlation estimator is equal to zero in the steady state, i.e., $E\left\{\widehat{r}_{m, \delta}(k)\right\}=0$

Proof: With respect to eqn (4) and considering the fact that $\left.\lim _{k \rightarrow \widehat{r}_{m, \delta}}(k)\right\}=E\left\{\widehat{r}_{m, \delta}(k-1)\right\}$, we obtain $E\left\{\widehat{r}_{m, \delta}(k)\right\}=E\left\{y_{l}(k) y_{m}^{*}(k-\delta)\right\}$. Two assumptions are introduced. (1) In the steady state, the $i^{\text {th }}$ source signal $s_{i}(k)$ is independent of the estimation error signal at the $j^{\text {th }}$ equalizer output, $e_{a}^{j}(k)$. This is actually a generalized assumption of that given in [2], whereby the case $i=j$ is specified. Justification of this assumption is based upon the fact that the estimation error of the equalizer- $j, e_{a}^{j}(k)$, is insensitive to the $i^{\text {th }}$ source signal. (2) The estimation error signals at the outputs of different equalizers are assumed uncorrelated, i.e., $E\left\{e_{a}^{i}(k) e_{a}^{j}(k-\delta)^{*}\right\}=0$ for $i \neq j$. This assumption becomes realistic when the equalizer is long enough so that its output in the steady state contains only an estimate of the corresponding source but no significant contribution from other interference sources. Under these two assumptions, by substituting $y_{i}(k)$ with the expression $s_{i}\left(k-d_{i}\right)-e_{a}^{i}(k)$ and utilizing the property of i.i.d. sources, the result $E\left\{\widehat{r}_{m, \delta}(k)\right\}=0$ is proven.

(b) When $k \rightarrow \infty, E\left\{\left|\widehat{r}_{m, \delta}(k)\right|^{2}\right\} \approx \frac{1-\lambda}{1+\lambda} E\left\{\left|s_{l}(k)\right|^{2}\left|s_{m}(k)\right|^{2}\right\}$

Proof: Expand the quantity $E\left\{\left|\widehat{r}_{m, \delta}(k)\right|^{2}\right\}$ with respect to eqn (4). In the resultant expansion, we assume the quantity $\widehat{r}_{m, \delta}(k-1)$ is uncorrelated with the equalizer output signals $y_{l}^{*}(k)$ and $y_{m}(k-\delta)$ when $\widehat{r}_{m, \delta}(k-1)$ converges to a constant in the steady state. This assumption is justified when the parameter $\lambda$ is chosen to be close to unity, which means a long data window is used in the cross-correlation estimation, [3]. Using the result of $E\left\{\widehat{r}_{m, \delta}(k-1)\right\}=0$ and for stationary sources $\lim _{k \rightarrow \infty} E\left\{\left|\widehat{r}_{m, \delta}(k)\right|^{2}\right\}=E\left\{\left|\widehat{r}_{m, \delta}(k-1)\right|^{2}\right\}$, the following approximation is achieved

$$
E\left\{\left|\widehat{r}_{m, \delta}(k)\right|^{2}\right\}=\frac{1-\lambda}{1+\lambda} E\left\{\left|s_{l}(k)\right|^{2}\left|s_{m}(k)\right|^{2}\right\}
$$

where the terms $\left|s_{m}(k-\delta)\right|^{2}\left|e_{a}^{l}(k)\right|^{2},\left|s_{l}(k)\right|^{2}\left|e_{a}^{m}(k-\delta)\right|^{2}$ and $\left|e_{a}^{l}(k)\right|^{2}\left|e_{a}^{m}(k-\delta)\right|^{2}$ are neglected for small values of $\left|e_{a}^{l}(k)\right|^{2}$ and $\left|e_{a}^{m}(k-\delta)\right|^{2}$.

Employing an energy preservation approach, the following relationship is derived in [2],

$\mu^{2} E\left\{\|\mathbf{x}(k)\|^{2}\right\} E\left\{\left|e_{o}^{l}(k)\right|^{2}\right\}=\mu E\left\{e_{a}^{l}(k)^{*} e_{o}^{l}(k)+e_{a}^{l}(k) e_{o}^{l}(k)^{*}\right\}$

Firstly consider $\mu^{2} E\left\{\left|e_{o}^{l}(k)\right|^{2}\right\}$ on the left hand side. Substituting the expression for $e_{o}^{l}(k)$, the expansion of $\mu^{2} E\left\{\left|e_{o}^{l}(k)\right|^{2}\right\}$ can be written as the sum of four terms.

$$
\mu^{2} E\left\{\left|e_{o}^{l}(k)\right|^{2}\right\}=\text { term } \mathrm{A}+\operatorname{termB}+\operatorname{term} \mathrm{C}+\operatorname{termD}(10)
$$

where termA $=\mu^{2} E\left\{\left|\left(R_{2}-\left|y_{l}(k)\right|^{2}\right) y_{l}(k)\right|^{2}\right\}$, termB $=\frac{\gamma^{2}}{4} \mu^{2} \operatorname{Tr}\left\{E\left\{\widehat{\mathbf{r}}(k) \widehat{\mathbf{r}}^{H}(k)\right\} E\left\{\mathbf{y}^{\prime}(k) \mathbf{y}^{\prime}(k)^{H}\right\}\right\}$, termC $=\frac{\gamma}{2} \mu^{2} E\left\{\left(\left|y_{l}(k)\right|^{2}-R_{2}\right) y_{l}^{*}(k)\left(\hat{\mathbf{r}}^{T}(k) \mathbf{y}^{\prime}(k)\right)\right\}$ and termD is the complex conjugate of termC. In [2], term $\mathrm{A}$ is approximated by $\mu^{2} E\left\{\left|s_{l}\right|^{2} R_{2}^{2}-2 R_{2}\left|s_{l}\right|^{4}+\left|s_{l}\right|^{6}\right\}$. To derive the expression for termB, on the basis that $\widehat{\mathbf{r}}(k)$ tends towards a vector with constant elements in the steady state, which is achieved by using a sufficiently long data window, 
we assume that matrices $\widehat{\mathbf{r}}(k) \widehat{\mathbf{r}}^{H}(k)$ and $\mathbf{y}^{\prime}(k) \mathbf{y}^{\prime}(k)^{H}$ are uncorrelated, [3]. Under this assumption, termB is written as term $\mathrm{B}=\frac{\gamma^{2}}{4} \mu^{2} \operatorname{Tr}\left\{E\left\{\widehat{\mathbf{r}}(k) \widehat{\mathbf{r}}^{H}(k)\right\} E\left\{\mathbf{y}^{\prime}(k) \mathbf{y}^{\prime}(k)^{H}\right\}\right\}$. Using the result of $E\left\{\left|\widehat{r}_{m, \delta}(k)\right|^{2}\right\}$ in eqn (8) and the statistical independence of the sources, term $B$ is approximated by $\frac{\gamma^{2}}{4} \mu^{2}(2 N+2 L+1) \frac{1-\lambda}{1+\lambda} E\left\{\left|s_{l}(k)\right|^{2}\right\} \sum_{m} E\left\{\left|s_{m}(k)\right|^{2}\right\}^{2}$, where terms which contain $\left|e_{o}^{l}(k)\right|^{2}$ are neglected for small value of $\left|e_{o}^{l}(k)\right|$. For termC, it is in fact the correlation between the instantaneous error signal contributed from the constant modulus cost and the decorrelation penalty. Hence, for small values of instantaneous error signals, termC can be approximated by zero. Since termD is the complex conjugate of termC, it is also approximately zero.

Refer to the right hand side of eqn (9). As the value of $\widehat{\mathbf{r}}(k)$ approaches to a constant vector in the steady state, the estimator of the cross-correlation between the current equalizer and the previous equalizer output $\widehat{\mathbf{r}}(k)$ is assumed to be uncorrelated with the product of estimation error $e_{a}^{l}(k)$ and the previous equalizer outputs $\mathbf{y}^{\prime}(k)$ in the steady state. Justification is also based upon $\widehat{\mathbf{r}}(k)$ approaching a vector with constant elements, when $\lambda$ is large, which corresponds to a long data window in the estimation of the cross-correlation penalty. Since $E\{\widehat{\mathbf{r}}(k)\}=\mathbf{0}$, the right hand side of eqn (9) can be written as $2 \mu E\left\{\rho\left|s_{l}(k)\right|^{2}\left|e_{a}^{l}(k)\right|^{2}-R_{2}\left|e_{a}^{l}(k)\right|^{2}\right\}$, where $\rho$ equals to 3 for a real system and 2 when the system is complex, [2]. Summarizing the above results and substituting the corresponding expression into eqn (9), the steady state MSE of the CC-CMA algorithm, i.e., $E\left\{\left|e_{a}^{l}(k)\right|^{2}\right\}$ when $k \rightarrow \infty$, is given by

$$
\begin{aligned}
& M S E=\mu E\left\{\|\mathbf{x}(k)\|^{2}\right\} \times \\
&\left.\frac{\left[\begin{array}{c}
E\left\{\left|s_{l}(k)\right|^{2} R_{2}^{2}-2 R_{2}\left|s_{l}(k)\right|^{4}+\left|s_{l}(k)\right|^{6}\right\}+ \\
\frac{\gamma^{2}}{4} \frac{1-\lambda}{1+\lambda}(2 N+2 L+1) E\left\{\left|s_{l}(k)\right|^{2}\right\}
\end{array}\right.}{\sum_{m} E\left\{\left|s_{m}(k)\right|^{2}\right\}^{2}}\right] \\
& 2\left[E\left\{\rho\left|s_{l}(k)\right|^{2}-R_{2}\right\}\right]
\end{aligned}
$$

When the mixing parameter $\gamma=0$, eqn (11) agrees with the results in [2] for the constant modulus algorithm. Eqn (11) shows that the CC-CMA steady state MSE is affected by the two-norm of the equalizer regressor $E\left\{\|\mathbf{x}(k)\|^{2}\right\}$, the step size $\mu$, the mixing parameter $\gamma$, the parameter $\lambda$, the number of i.i.d. sources and the source statistics. Large values of $\mu$ and $\gamma$ lead to high level of MSE. However, with small $\mu$, convergence rate reduces and there exists a lower bound for the mixing parameter $\gamma$, [4]. For a system with a larger number of sources, the steady state MSE increases, which is reasonable since the adaptation of the current equalizer is related to the output of the previous equalizer due to the cross- correlation penalty and the error of the previous equalizer output would propagate to the equalizer at the later stage.

\section{THE MODIFIED CC-CMA ALGORITHM}

When compared with constant modulus sources, open eye patterns are more difficult to achieve for non-constant modulus sources due to the high level of steady state MSE. To overcome this problem, the cost function of the modified CC-CMA algorithm (MCC-CMA) for the $l^{\text {th }}$ equalizer is written as

$$
J_{l}^{\text {new }}(k)=J_{l}(k)+\gamma_{2} E\{p(k)\}
$$

where $\gamma_{2}$ is an additional mixing parameter to combine a constellation matched error penalty together with the conventional cost function. The term $E\{p(k)\}$ is newly introduced to reduce the constellation matched error. Notice that in the selection of the function $p(k)$, the following factors should be considered [5]. Firstly, $p(k)$ should provide uniform performance over the information symbols. Secondly, it should be symmetric around each member of the alphabet. Thirdly, the maximum value should be reached at the centre point between two consecutive symbols from the alphabet. The minimum values are zeros and only occur at the constellation points. For a PAM-PSK signal constellation, a similar constellation matched error function to that in [5] is employed. That is,

$$
p(k)=1-\sin ^{2 n}\left(\frac{\left|y_{l}(k)\right|}{2 d} \pi\right)
$$

where $2 d$ is the minimum distance between symbols and $n$ is a positive integer which gives some degree of freedom for the selection of $p(k)$. Using the stochastic gradient method to minimize the MCC-CMA cost function, the instantaneous error signal is modified so that the update equation of the MCC-CMA algorithm at the $k^{\text {th }}$ iteration is written as

$e_{o}^{l}(k)_{\text {new }}=\left[\begin{array}{c}\left(R_{2}-\left|y_{l}(k)\right|^{2}\right) y_{l}(k)-\frac{\gamma}{2} \widehat{\mathbf{r}}^{T}(k) \mathbf{y}^{\prime}(k)+ \\ \frac{\gamma_{2} n \pi}{d \mid y_{l}(k) !} \sin ^{2 n-1}\left(\frac{\left|y_{l}(k)\right|}{2 d} \pi\right) \cos \left(\frac{\left|y_{l}(k)\right|}{2 d} \pi\right) y_{l}(k)\end{array}\right]$

The performance of the newly proposed modified MCCCMA algorithm is examined in the following simulation section.

\section{SIMULATIONS}

Firstly we examine the steady state MSE of the CC-CMA algorithm. A MIMO system with three i.i.d. sources and four sensors is assumed. The order of the channel and the sub-equalizer are respectively $L=1$ and $N=2$. We assume a QPSK system with source alphabet $\left\{ \pm \frac{1}{\sqrt{2}} \pm \frac{1}{\sqrt{2}} j\right\}$ 

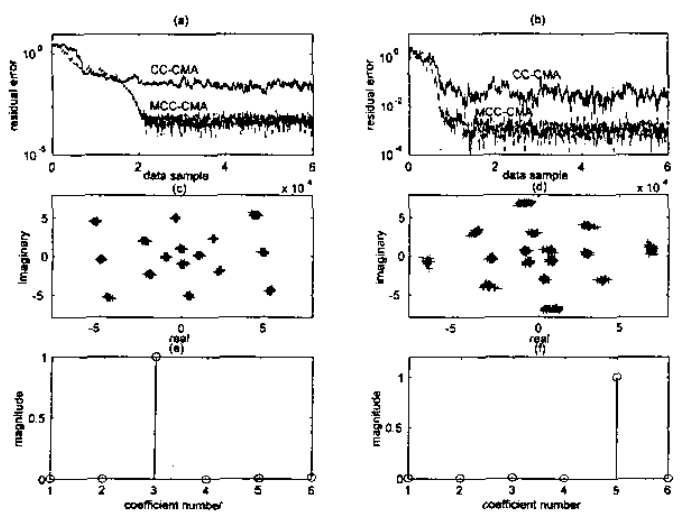

Fig. 1. Comparison between CC-CMA and MCC-CMA. (a) Residual error at EQ-1 (b) Residual error at EQ-2 (c) Eye diagram of EQ-1 after 20000 samples, MCC-CMA (d) Eye diagram of EQ-2 after 20000 samples, MCC-CMA (e) Combined channel plus EQ-1 response (f) Combined channel plus EQ-2 response

for a constant modulus source. For the non-constant modulus source, an 8-PAM-PSK constellation is employed, page 179 [6]. Fixing the parameters $\lambda=0.999$ and $\gamma=4$, the experimental results are shown in Table (1). Fairly close agreement between the experimental result and analysis can be observed. Note that the step size $\mu$ for equalizer-1, 2,3 are respectively chosen as $3 \times 10^{-3}, 2 \times 10^{-3}$ and $0.6 \times 10^{-3}$ for the QPSK system and $3 \times 10^{-3}, 5 \times 10^{-5}$ and $5 \times 10^{-5}$ for the 8-PAM-PSK system. Simulations indicate that the steady state MSE for the non-constant modulus sources are much larger than that of the constant modulus sources even though much smaller step sizes are used. More simulation results regarding varying value of $\lambda, \gamma$ and $\mu$ are shown in [3]. The performance of the MCC-CMA algorithm is next examined. A two users and three sensors 16-PAMPSK system is assumed. Let $L=N=1$ and set the degree $n=1$. The conventional CC-CMA algorithm and the MCC-CMA algorithm are compared in Fig 1(a) and (b) in terms of residual error. Analogous to the steady state MSE, the residual error for the $l^{\text {th }}$. equalizer is defined as $\frac{\left\|\mathbf{h}_{l}(k)\right\|_{2}^{2}-\max \left(\left|\mathbf{h}_{l}(k)\right|\right)^{2}}{\max \left(\mathbf{h}_{l}(k) \mid\right)^{2}}$ and is an important measure in indicating when the open eye condition can be achieved. With the MCC-CMA algorithm, both equalizer- 1 and equalizer- 2 achieve open eye patterns within 20,000 data samples (compared with more than 60000 data samples with the CCCMA algorithm). The combined channel plus equalizer-1 and equalizer-2 impulse responses are shown in Fig 1(e) and (f). It can be observed that equalizer-1 retrieves source-1 with two delays and equalizer- 2 reconstructs source- 2 also with two delays.

\begin{tabular}{|l|l|l|l|l|}
\hline & & Equalizer-1 & Equalizer-2 & Equalizer-3 \\
\hline QPSK & Ana. & 0 & $4.72 \times 10^{-4}$ & $2.83 \times 10^{-4}$ \\
& Sim. & $1.40 \times 10^{-20}$ & $0.33 \times 10^{-4}$ & $0.55 \times 10^{-4}$ \\
\hline 8-PAM & Ana. & $0.95 \times 10^{-1}$ & $1.01 \times 10^{-1}$ & $0.64 \times 10^{-1}$ \\
-PSK & Sim. & $2.21 \times 10^{-1}$ & $1.81 \times 10^{-1}$ & $1.24 \times 10^{-2}$ \\
\hline
\end{tabular}

Table 1. MSE signal at the output of equalizer-1, 2, 3 for the QPSK and 8-PAM-PSK systems

\section{CONCLUSION}

An analytical expression for the steady state MSE of the CC-CMA algorithm is derived. Its value is proportional to the step size $\mu$ and the quantity of $E\left\{\|\mathbf{x}(k)\|^{2}\right\}$ and is furthermore affected by the choice of the mixing parameter $\gamma$, the parameter $\lambda$, which controls the window length within the estimation of the cross-correlation penalty, the source statistics and the number of users in the system. To reduce the steady state MSE when the signal constellation is not constant modulus, an addition constellation matched error penalty is introduced, resulting in the new modified CCCMA algorithm. Simulation results support the analysis and confirm the improvement of the MCC-CMA algorithm.

\section{REFERENCES}

[1] C.B. Papadias and A. Paulraj, "A constant modulus algorithm for multiuser signal separation in presence of delay spread using antenna arrays", IEEE Signal Processing Letters, vol. 4, no. 6, pp. 178-181, 1997.

[2] J. Mai and A. H. Sayed, "A feedback approach to the steady-state performance of fractionally spaced blind adaptive equalizers", IEEE Trans. on Signal Processing, vol. 48, no. 1, pp. 80-91, 2000.

[3] Y. Luo and J. A. Chambers, "Steady state meansquare error analysis of the cross-correlation and constant modulus algorithm in a MIMO convolutive system", Accepted by IEE Proceedings - Vision, Image and Signal Processing, 2002.

[4] A. Touzni, I. Fijalkow, M.G. Larimore, and J.R. Treichler, "A globally convergent approach for blind MIMO adaptive deconvolution", IEEE Trans. on Signal Processing, vol. 49, no. 6, pp. 1166-1178, 2001.

[5] M. Amin, L. He, C. Reed Jr, and R. Malkemes, "A modified constant modulus algorithm for adaptive channel equalization for QAM signals", in Proc. Of the IEEE Signal Processing Workshop, Statistical Signal Processing, 2001, pp. 563-566.

[6] J. G. Proakis, Digital Communications, McGraw Hill, 1995. 